\title{
Interplanetary Magnetic-Sector Structure, 1926-1971
}

\author{
L. SvalgaARD ${ }^{1}$ \\ Danish Meteorological Institute, Geophysical Section, Copenhagen, Denmark
}

\begin{abstract}
The influence of the direction of the interplanetary magnetic field on the geomagnetic field at high latitudes is used to study the long-term behavior of the sector structure during nearly four solar cycles. It is found that the rotation period of the sector structure varies from about 28.5 days in the beginning of a solar cycle to 27.0 days in the end. Also it is shown that short-lived sectors rotate more slowly than long-lived ones.
\end{abstract}

Geomagnetic effects related to the interplanetary magnetic field. A relation between the polarity of the interplanetary magnetic field and the type of diurnal variation of the geomagnetic field inside the polar cap has been discovered independently by Svalgaard [1968], Mansurov [1969], and Mansurov and Mansurova [1970] and noted by Iwasaki [1971]. The relationship has been confirmed by FriisChristensen et al. [1971]. The most pronounced effect, a broad perturbation of the geomagnetic field lasting for several hours, is found in the vertical component $Z$ of the field on stations near the invariant poles. Figure 1 shows magnetograms from the near-conjugate stations Thule (86.8 $8^{\circ}$ invariant latitude) and Vostok $\left(-84.9^{\circ}\right.$ invariant latitude). To increase readability, all magnetograms in this paper have been redrawn on the basis of hourly mean values. When the earth lies in a polarity sector of the interplanetary magnetic field away from the sun, the $Z$ perturbation is directed away from the earth; in a sector toward the sun the perturbation is directed toward the earth in both hemispheres. As is seen from the magnetograms, it is easy by visual inspection to distinguish two types of disturbances. This is the basis of the following simple classification scheme.

A day is classified as type $C$ if the $Z$ magnetograms from the near-pole stations for that day show a broad positive perturbation between magnetic noon and local noon; a negative

1 Now at Institute for Plasma Research, Stanford University, Stanford, California 94305.

Copyright @ 1972 by the American Geophysical Union. perturbation classifies the day as type A. An observer using magnetograms from a southern polar-cap station should adopt the definition that a positive $Z$ perturbation is directed toward the earth. In general, the regularity of the magnetograms leaves little doubt about the type of perturbation. With very few exceptions it is possible to classify every day. Figure 2 shows some sequences of $Z$ records from Resolute Bay ( $84.3^{\circ}$ invariant latitude). It is clearly seen that the same type persists through several days. In some of the sequences a change of type is demonstrated.

This classification was actually developed be-
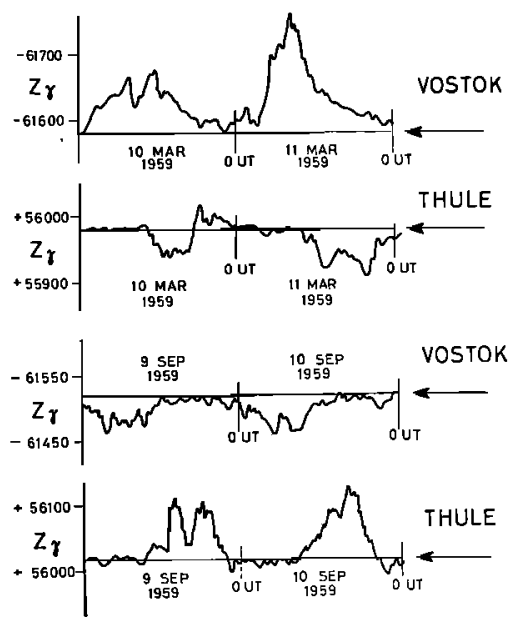

Fig. 1. Magnetograms showing the geomagnetic effects of the sectoring of the interplanetary magnetic field in the sector away from the sun (top) and in sector toward the sun (bottom) at Thule $\left(86.8^{\circ}\right.$ invariant latitude) and Vostok $\left(-84.9^{\circ}\right.$ invariant latitude). The quiet undisturbed fields are indicated by arrows. 

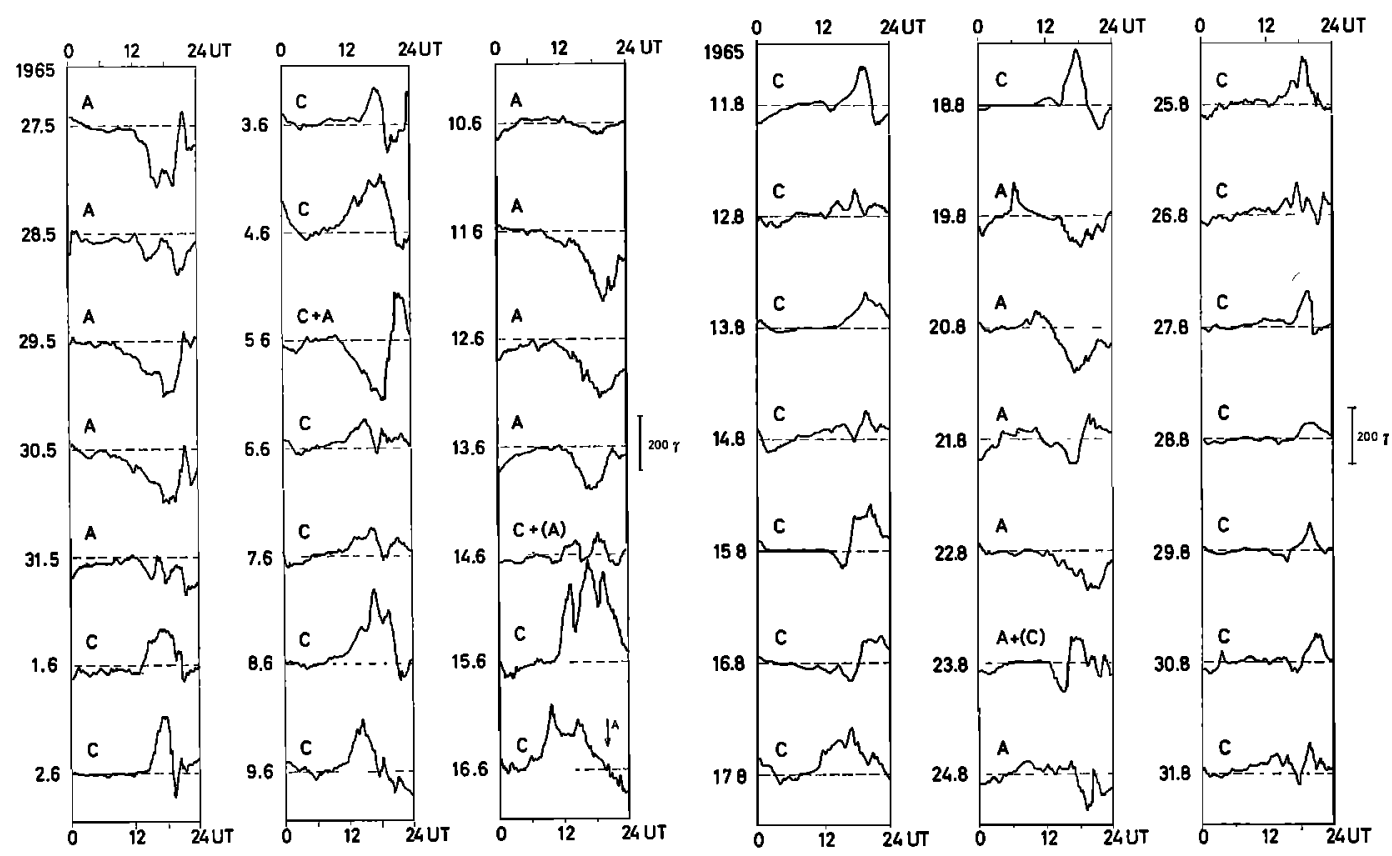

Fig. 2. Series of consecutive records of the vertical component $Z$ of the geomagnetic field at Resolute Bay. The notation 27.5 means May 27, 28.5 is May 28, and so forth.

fore the relationship with the sector structure was discovered. When the sector structure of the interplanetary magnetic field was discovered [Wilcox and Ness, 1965], it became evident that A variations dominated during away sectors, whereas $\mathrm{C}$ variations were found during toward sectors.

Figure 3 compares the observed sector structure with the A-C classification as derived from Canadian IQSY stations for 1964-1965. This classification uses the $Z$ records from stations with an invariant latitude of about $85^{\circ}$. The $Z$ perturbations decrease with increasing distance from the invariant poles. Instead they turn into a perturbation of the horizontal component $H$. For a station such as Godhavn (77.5 ${ }^{\circ}$ invariant latitude) the $H$ component increases on $\mathrm{A}$ days and decreases on $\mathrm{C}$ days. Figure 4 shows the relation between deviations of daily mean values from the monthly mean for $Z$ at Thule and $H$ at Godhavn. The relation is so close that the $H$ magnetograms from Godhavn may as well be used to infer the type of day. As a matter of fact, during the winter months it is often easier to base the classification work on Godhavn $H$ records than to base it on Thule $Z$ records. The magnetic observatory at Godhavn has been operating continuously since 1926 . This unique series of magnetograms is the basis of the investigation of the long-term behavior of the sector structure presented in the last part of this paper.

Sector polarity since 1926. A table presenting the result of an A-C classification of each day since February 1, 1926, has been prepared, but because of its great length only the last 10 years are given in this paper; the complete table may be obtained in machine readable form from the author. The full table is also published by Svalgaard [1972]. Several important conclusions may be drawn from this material. An autocorrelation analysis of the class of the day during the whole period 1926-1970 is illustrated by Figure 5. A recurrence property is clearly evident.

The recurrence peaks have been labeled with the number of recurrence periods that they represent. The recurrence period may be interpreted as the average rotation period of the sector structure. In Figure 6 it seems that the more stable the recurrence (high peak numbers) is, the faster the pattern rotates. In other words, short-lived sectors rotate more slowly than longlived ones. A similar effect has been discovered in an analysis of the interplanetary magnetic field observed by spacecraft [Wilcox and Tanen- 


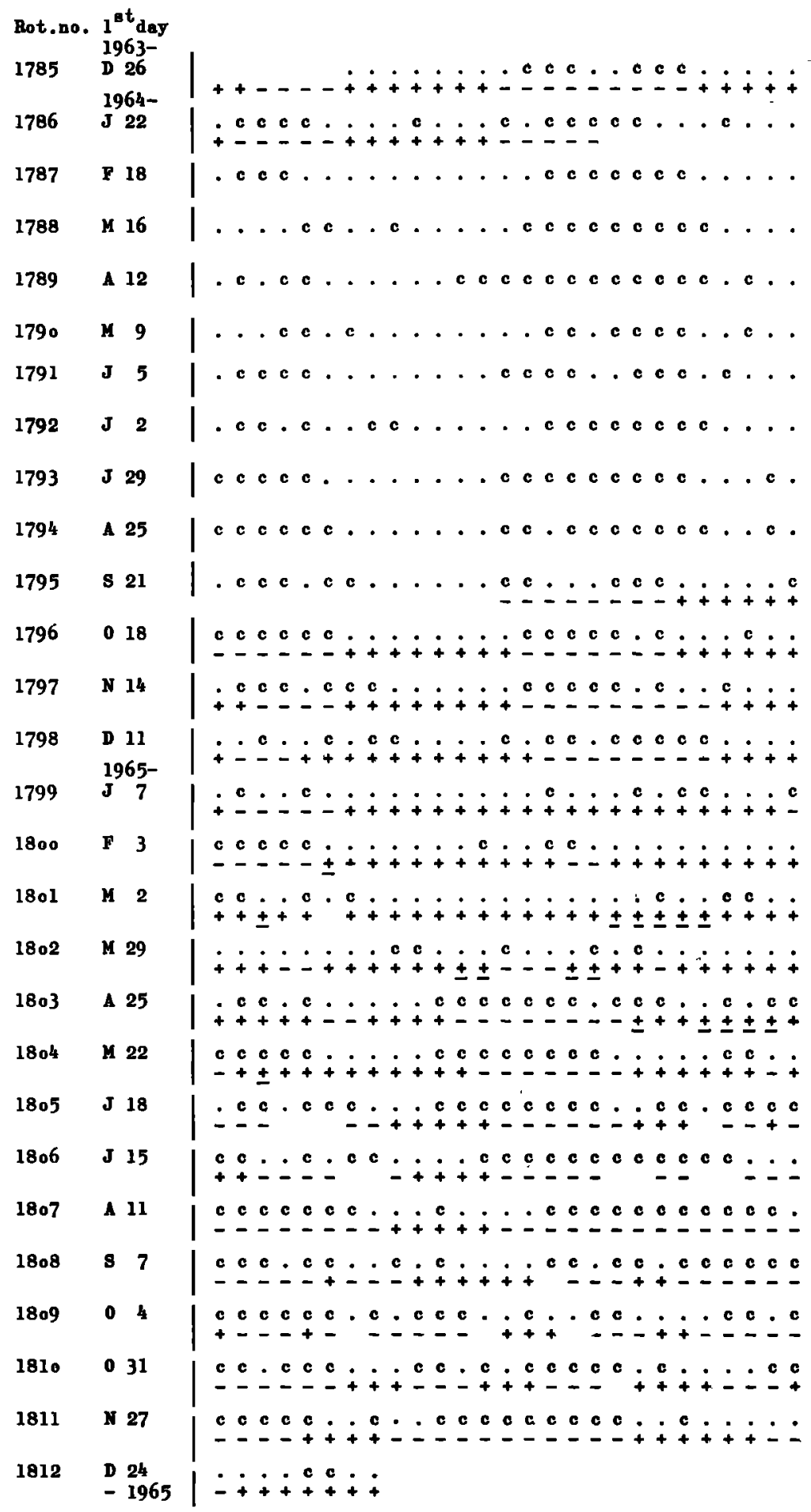

Fig. 3. Comparison of the observed sector structure with the A-C classification. Plus indicates interplanetary magnetic field directed away from the sun, minus indicates field directed toward the sun, dots indicate type $A$ daily variations, and $c$ indicates type $C$ variations of the vertical component $Z$. The data are ordered in sun rotation periods. (Reproduced from Svalgaard [1968].) 
TABLE 1. Negative (A) and Positive (C) Perturbations for 1962-1971

\begin{tabular}{|c|c|c|c|c|c|c|c|}
\hline Month & & & Clas & ation by I & & & \\
\hline $\begin{array}{c}1962 \\
\text { January }\end{array}$ & AAAAA & $\mathrm{ACCCC}$ & CAACC & CAACA & AAAAA & AAAAA & A \\
\hline February & AAACC & CCAAA & CCCCC & CAAAA & AAACA & $\mathrm{CAA}$ & \\
\hline March & ACCAC & CAAAC & CCAAA & AAAAC & CAACC & AAAAC & A \\
\hline April & AACAA & CCCCC & CCAAA & AAAAA & ACAAC & ACCCC & \\
\hline May & CCAAA & CAAAA & CAAAC & CAAAA & AAAAA & ACCAA & C \\
\hline June & ACCCC & CAAAA & AAAAA & AAAAA & CACCC & CCCCC & \\
\hline July & AAAAA & ACAAA & AACAC & AAAAC & CAACA & CCCCA & A \\
\hline August & AAAAA & ACAAA & AAACC & CCCCA & ACCCC & AAAAA & C \\
\hline September & AAACC & AAACC & CCCCC & AAACA & CCACC & AAAAA & \\
\hline October & CCAAA & AACCC & CCCCA & CACCC & ACCCA & CACCC & $\mathbf{A}$ \\
\hline November & ACACA & CCCAA & CAACC & CAAAA & CCCAC & AAAAA & \\
\hline $\begin{array}{c}\text { December } \\
1963\end{array}$ & AAACC & $\mathrm{ACCCC}$ & CCCCC & $\mathrm{ACCCC}$ & CCAAA & CAAAA & C \\
\hline January & CAACC & CCCCC & CCCAA & CAACA & AAAAA & AAAAC & $\mathbf{C}$ \\
\hline February & CCAAA & CCCAA & ACCAC & AAAAA & AAAAA & AAA & \\
\hline March & ACACC & CCCCA & CAAAC & $\mathrm{ACCCC}$ & ACAAC & AAACA & $\mathbf{A}$ \\
\hline April & CAAAA & AAAAC & CCCCC & CCCAA & ACCCA & ACAAC & \\
\hline May & AACCA & AACCC & CCACA & AAAAC & CACAC & CCAAA & A \\
\hline June & AAAAC & ACCCC & ACCAA & CCCAC & A'AACA & AAAAC & \\
\hline July & AAACC & CCACC & ACACC & ACACC & AAAAA & AAAAC & $\mathrm{C}$ \\
\hline August & CCCAC & ACACA & ACCCC & CCAAA & ACAAC & CCCCA & $\mathbf{A}$ \\
\hline September ' & CACCC & ACCCC & ACCAA & AAAAA & CCACC & CCACC & \\
\hline October & AAACC & ACCAA & AAAAA & AAAAA & ACCCA & CAAAC & $\mathbf{C}$ \\
\hline November & CCCCA & CCAAA & AAAAA & ACCCA & CCACC & CAAAC & \\
\hline $\begin{array}{c}\text { December } \\
1964\end{array}$ & CCCCC & AAAAA & AAACC & CCCCC & ACCCA & AACCC & C \\
\hline January & AAAAA & AAACC & CAACC & CAAAA & AACCC & CAAAA & C \\
\hline February & AAACA & CCCCC & AAACA & AAACC & CAAAA & AAAA & \\
\hline March & AAACC & CCCCC & AAAAA & AAAAC & CAACA & AAAAC & C \\
\hline April & CCCCC & CCAAA & AACAC & CAAAA & AACCC & $\mathrm{CCCCC}$ & \\
\hline May & CCCCA & CAAAA & ACCAC & AAAAA & AAACC & ACCCC & $\mathbf{A}$ \\
\hline June & ACAAA & CCCCA & AAAAA & $\mathrm{AACCC}$ & CAACC & CACAA & \\
\hline July & AACCA & CAACC & AAAAA & ACCCC & CCCCA & AAACC & C \\
\hline August & CCAAA & AAAAA & CCCCC & CCCCA & AACAC & CCCCC & A \\
\hline September & AAAAA & ACCAC & CCCCC & CAACA & ACCCA & CCAAA & \\
\hline October & AAACC & AAACC & CAAAA & ACCCC & CCCAA & AAAAA & A \\
\hline November & CCCCC & ACAAA & CAAAC & CCACC & CAAAA & AACCC & \\
\hline $\begin{array}{c}\text { December } \\
1965\end{array}$ & CCACA & ACAAA & AACAA & CACCA & AAACA & CCACC & C \\
\hline January & CCAAA & AACAA & CAAAA & AAAAA & ACAAA & CACCA & $\mathbf{A}$ \\
\hline February & ACCCC & CCAAA & AAAAC & AACCA & AAAAA & $\mathrm{AAA}$ & \\
\hline March & ACCAA & CACAA & AAAAA & AAAAA & ACAAC & CAAAA & A \\
\hline April & AAAAA & CCAAA & CAAAC & ACAAA & AAAAA & CCACA & \\
\hline May & AAAAC & CCCCC & CACCC & AACAC & CCCCC & CAAAA & A \\
\hline June & CCCCC & CCCAA & AAACC & AAACC & ACCCA & AACCC & \\
\hline July & CCCCC & AACCA & CCCCC & CAACA & CCAAA & ACCCC & C \\
\hline August & CCCCC & CCAAA & CCCCC & CCAAA & CAAAA & CCCCC & $\mathrm{C}$ \\
\hline September & CCCCC & ACCCA & CCAAC & ACAAA & ACCAC & CACCC & \\
\hline October & CCCCC & CCCCA & CACCC & AACAA & CCAAA & ACCAC & $\mathbf{C}$ \\
\hline November & CACCC & AAACC & ACACC & CCCAC & AAAAC & CCCCC & \\
\hline $\begin{array}{c}\text { December } \\
1966\end{array}$ & CAACA & ACCCC & CCCCC & AACAA & AAAAA & AACCA & $\mathbf{A}$ \\
\hline January & AAAAA & AACAC & ACAAA & AACCC & CCCCC & AAAAA & $\mathbf{A}$ \\
\hline February & AAAAC & AAAAC & CCCAA & AAAAA & CAAAA & AAA & \\
\hline March & AAACC & CCAAA & CACCA & ACAAA & CACCA & AAAAC & C \\
\hline April & CCACC & CCAAA & AAACA & CAAAA & CAACC & AAACA & \\
\hline May & CCCCA & AAAAC & AAAAA & CCCCC & AAAAA & ACACC & C \\
\hline June & CCCAA & AACCC & CCCAC & AAACA & CACCC & CCCCC & \\
\hline July & CCCCA & AACAC & CCAAC & CAAAC & CCCCA & CCACC & C \\
\hline Aligust & AAAAC & CACCA & AAAAA & AACCC & CCCAC & CCCCC & $\mathrm{C}$ \\
\hline September & ACCAC & AAAAA & AAACC & CCCCA & CCCCC & CCCCA & \\
\hline October & AACAA & AAAAA & CCCCC & ACCCC & CACCC & CCCCC & A \\
\hline
\end{tabular}


TABLE 1. (continued)

\begin{tabular}{|c|c|c|c|c|c|c|c|}
\hline \multirow{3}{*}{$\begin{array}{c}\text { Month } \\
\text { November } \\
\text { December } \\
1967\end{array}$} & \multicolumn{7}{|c|}{ Classification by Day } \\
\hline & CAAAA & CACCA & CCCCA & CCCCA & CCCAA & CCAAA & \\
\hline & AAACA & CACCC & ACCCA & CAAAC & CAAAA & ACAAA & C \\
\hline January & CCCAA & AACAC & $\mathrm{CCCCC}$ & CCAAA & CAAAA & AACCC & A \\
\hline February & AAAAC & ACCCC & CCCAC & AAAAA & ACAAA & AAC & \\
\hline March & AAAAA & $\mathrm{ACCCC}$ & CAACA & AAAAC & CCAAA & AACAA & A \\
\hline April & AACCA & ACACC & CCAAA & CCCCC & CCAAA & AAAAA & \\
\hline May & AACCC & AAACC & CCCCC & CCACC & ACAAA & CCACC & C \\
\hline June & CCAAA & CCCAC & AACCC & ACCAC & CCCAC & CCCCA & \\
\hline July & ACAAA & AACCC & CCCCC & ACCAC & ACCCC & CCCAA & A \\
\hline August & $\mathrm{ACCCC}$ & AAACC & CCCCA & CCCCC & CCAAC & CAACC & A \\
\hline September & AAAAA & CCCCC & CCCCA & CCCAA & CCCCC & AAAAA & \\
\hline October & ACCCA & ACAAC & CCCCC & CACCC & CAAAA & ACCAC & C \\
\hline November & AACAA & AACCC & CCACA & CCCCA & CCCCA & ACCAA & \\
\hline $\begin{array}{c}\text { December } \\
1968\end{array}$ & CCAAC & AACCC & AAACA & CCCCC & AAAAC & CAAAC & C \\
\hline January & CAACC & CACCC & CAAAC & AAAAA & AAACC & AAACA & $\mathbf{C}$ \\
\hline February & CCCAC & CAAAA & AAAAA & CCCCC & AAAAA & $\mathrm{ACCC}$ & \\
\hline March & CCCCC & CCCAA & AAAAC & AAACC & ACCCC & CCAAC & C \\
\hline April & CCACA & AAACC & AAAAC & ACAAC & CCCCA & CCCAC & \\
\hline May & AAAAA & CCAAA & AACCC & CCCCC & CCCCC & CCAAA & C \\
\hline June & CCAAC & AAACA & CACCC & CCCCA & CCAAA & $\mathrm{ACCCC}$ & \\
\hline July & CAAAA & AAACC & CCCCC & AAAAA & AAACC & CACCC & A \\
\hline August & AAACC & CCAAA & CAACA & AAACC & CCCCA & CCCCA & A \\
\hline September & CCACC & CAAAA & AAAAA & AAACC & CCCCA & AAAAC & \\
\hline October & AACCC & AAAAC & AAAAA & ACACC & CCCCC & AAACC & $\mathbf{C}$ \\
\hline November & CACAA & AAAAA & ACACC & CCCCC & CACAA & ACCAC & \\
\hline December & CCCCA & $\mathbf{A A A A C}$ & AAAAC & CCCCA & CAAAA & AAAAA & C \\
\hline 1969 & & & & & & & \\
\hline January & CCCCC & CCCCC & CCCAC & CCAAA & AAAAA & CAAAA & A \\
\hline February & CCCCC & CCACC & CCCCA & AAAAA & AAAAA & AAA & \\
\hline March & AAACC & CCCCC & CCCCC & CACCC & CCCCA & AAAAA & $\mathbf{A}$ \\
\hline April & $\mathrm{ACCCC}$ & CCCCA & ACCAC & CCACA & AACAA & AACCC & \\
\hline May & CCCCC & CAAAA & ACCCA & CCAAA & AAAAA & ACACC & $\mathrm{C}$ \\
\hline June & CCCCC & AACCA & ACAAA & AAAAA & ACCCC & CCCAC & \\
\hline July & CCACA & CCCCA & AAAAA & AACAA & CCCCC & CCCAA & A \\
\hline August & CCCAC & CAAAA & AAAAA & CCCCC & CCCCC & AACAC & C \\
\hline September & CCCAA & ACCAA & AAAAA & AACCC & CACCC & CCCCA & \\
\hline October & AAAAA & AAAAA & AAAAC & CCCCC & CCCCC & CCAAA & A \\
\hline November & AAAAA & ACAAC & CCCCC & CCACC & CCCCC & ACACC & \\
\hline December & AAAAA & CCCCA & CCCCC & CCCCA & CACAC & CAAAA & $\mathbf{A}$ \\
\hline 1970 & & & & & & & \\
\hline January & ACAAC & CCCCA & ACCCC & CCCAA & AAACA & AACCC & A \\
\hline February & AACCC & CAACA & ACCCA & CCAAA & AACAA & AAC & \\
\hline March & CCCAC & AACAA & CCACA & AAAAA & ACAAA & AAAAC & $\mathbf{C}$ \\
\hline April & AAACA & ACAAA & AAACA & CCCAA & ACCCC & CCCCA & \\
\hline May & AAAAA & AAAAC & ACCCC & CAAAC & CCCCC & CCAAA & $\mathbf{A}$ \\
\hline June & CCAAC & AACCC & CCCCC & CCACC & CACAA & ACAAC & \\
\hline July & AAAAC & CCCAA & $\mathrm{CCCCC}$ & CCAAC & ACAAA & ACCAC & C \\
\hline August & CCCCA & ACCCC & CCCCC & ACAAC & AAAAA & AAAAA & A \\
\hline September & CCCAC & CCCCC & CAAAA & AAAAA & AAAAA & AAACC & \\
\hline October & CCCAC & CCCCC & AAAAA & AAAAA & CAACA & ACACC & C \\
\hline November & CCCCA & ACCAA & AAAAA & ACCAA & AACCC & AACAC & \\
\hline December & CCCCA & CAAAC & AAACC & AAAAA & AACCC & CCCCA & A \\
\hline 1971 & & & & & & & \\
\hline January & AAAAA & ACCCC & AAAAA & AACAC & CCCCC & AAACC & C \\
\hline February & CACCC & CAAAA & AAACC & CCCCA & CCACA & CAA & \\
\hline March & ACACC & CAAAA & AACCC & ACCCC & CCAAA & CAAAA & C \\
\hline April & CAAAC & AAACC & CCCCA & ACAAA & AACAA & ACCAA & \\
\hline May & AAACA & CACCC & CCCCC & AAAAA & ACACC & CCCAA & A \\
\hline June & CCCCC & $\mathrm{CACCC}$ & CACAC & AAACA & AAACA & AAACC & \\
\hline
\end{tabular}




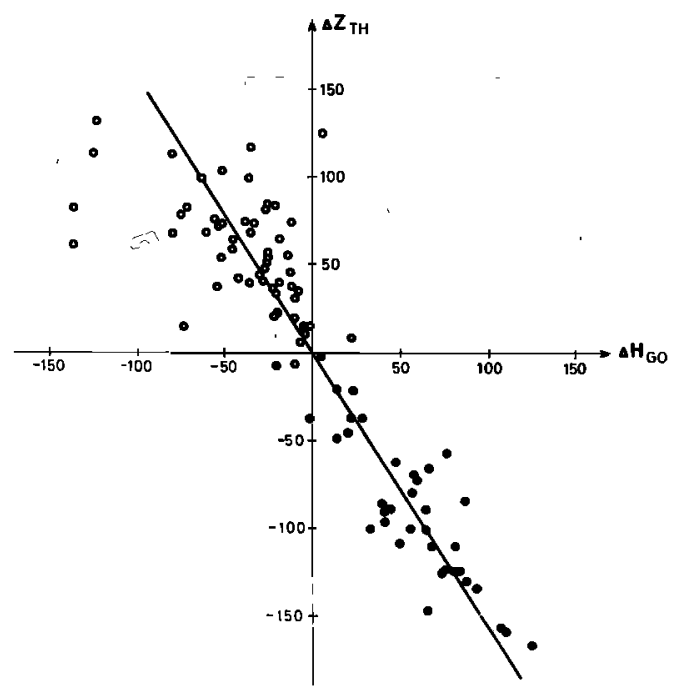

Fig. 4. Relationship between deviations of daily mean values from the monthly mean for $Z$ at Thule and $H$ at Godhavn. Data points are from June to August 1959. Solid circles denote days within inferred sectors away from the sun, and open circles days within inferred sectors toward the sun.

baum, 1971] and in an analysis of the photospheric magnetic field [Wilcox et al., 1970].

To examine the variation of the average

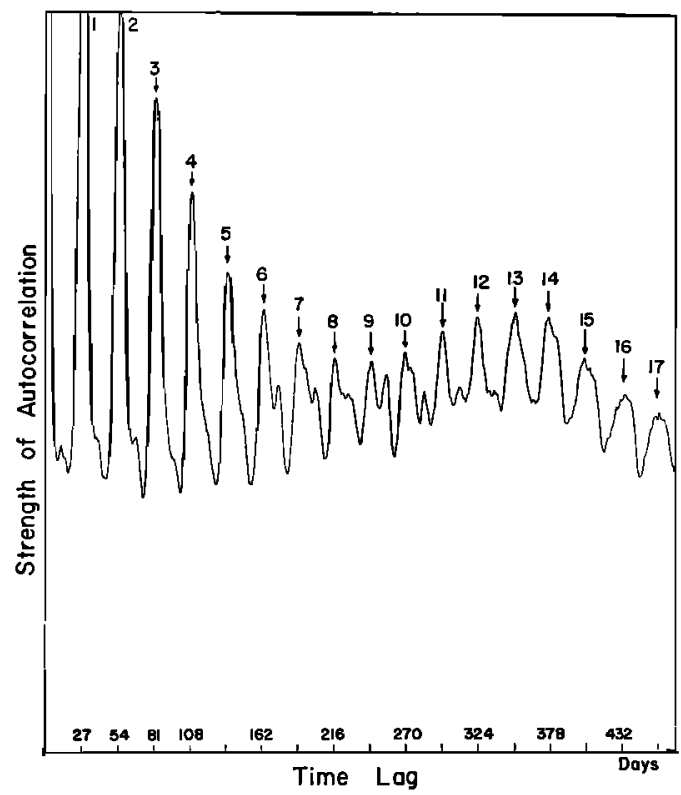

Fig. 5. Autocorrelation of the direction of the inferred interplanetary magnetic field, 1926-1971.

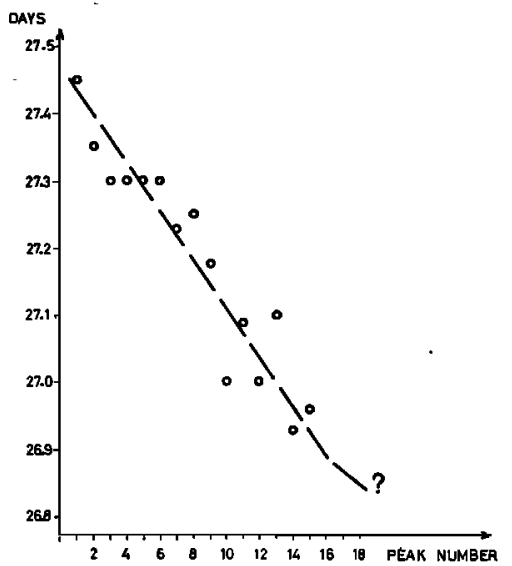

Fig. 6. Recurrence period for the direction of the interplanetary magnetic field compared with the number of the recurrence peak.

period of the sector structure through the sunspot cycle, autocorrelations were computed for each year from 1926 to 1970 . The observation that years with the same phase within the solar cycle have very similar autocorrelation curves then justifies computing an average autocorrelation curve for all years having the same relative position within the sunspot cycle. This set

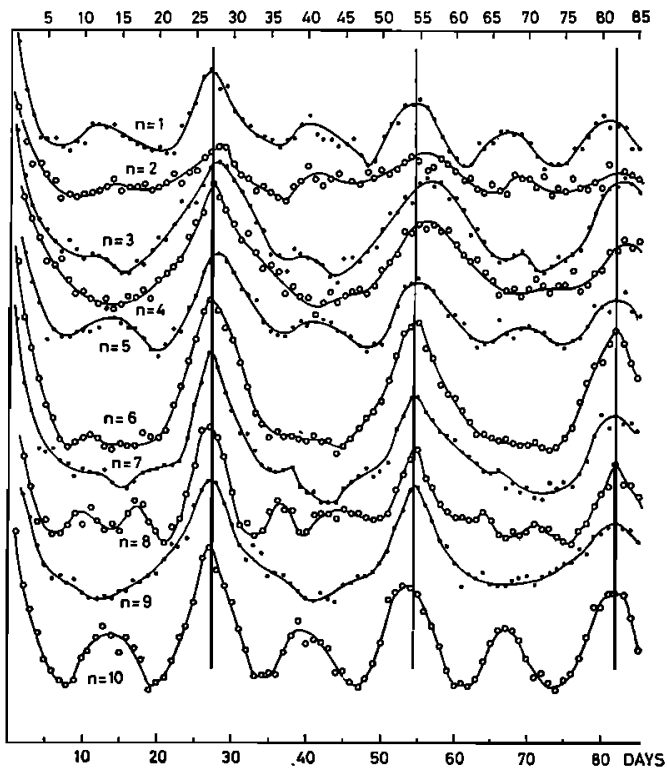

Fig. 7. Autocorrelations of the direction of the inferred interplanetary magnetic field for different years in the sunspot cycle. The number $n$ denotes years following sunspot minimum. 


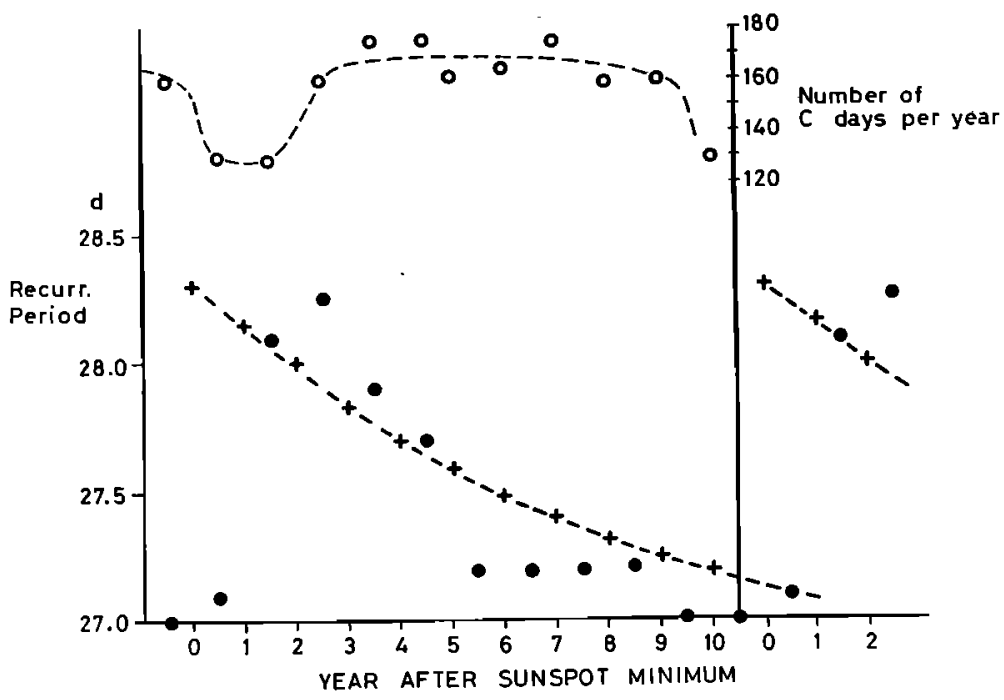

Fig. 8. Average recurrence period for the sector structure (solid circles) during the solar cycle. Also shown are the average rotation period for sunspots (crosses) and the number of $\mathrm{C}$ days, or polarity toward the sun, per year (open circles).

of curves is shown in Figure 7. The recurrence period shows a systematic variation through the cycle (Figure 8). Near sunspot minimum the period is 27.1 days. With the rise of the new high-latitude solar activity the recurrence period increases sharply to 28.3 days; with the progress of the cycle the period decreases and remains rather constant at about 27.2 days, during the last half of the cycle. The variation of the recurrence period resembles the variation of the rotation period of sunspots through the solar cycle.

Just around sunspot minimum the number of $\mathrm{C}$ days per year (days with the probability of a sector toward the sun) is significantly smaller than that during the rest of the cycle. Often it seems as if the $\mathbf{C}$ sectors are dying away just at minimum. When the new activity starts, it begins rather erratically with no well-developed sector structure. After sunspot maximum the sector structure is usually well defined before it disappears again near the next minimum. The disappearance usually happens within one or two solar rotations and may provide an independent way of determining the time of the beginning of the next cycle. Also near minimum there is a high probability for the occurrence of a four-sector structure, as is seen from the secondary peaks in Figure 7 for $n=1$ and $n=10$.

Table 1 includes a prediction of the sector polarity during 1970 and the first half of 1971 . This prediction will make possible a strong test of the connection between the A-C effects and the sector polarity in the sense that the structure predicted is not just an extrapolation of that of 1969 but includes the disappearance of a sector and the creation of a new one having a different phase.

Acknowledgments. I would like to thank the staff of the Geophysical Section of the Danish Meteorological Institute, headed by $K$. Lassen, for use of data from the observatories in Greenland and for helpful discussions on the subject.

The Editor thanks S.-I. Akasofu, S. Matsushita, and J. M. Wilcox for their assistance in evaluating this paper.

\section{REFERENCES}

Friis-Christensen, E., K. Lassen, J. M. Wilcox, W. Gonzalez, and D. S. Colburn, Interplanetary magnetic sector polarity from polar geomagnetic field observations, Nature, 238, 48, 1971.

Iwasaki, N., Localized abnormal geomagnetic disturbance near the geomagnetic pole and simultaneous ionospheric variations, Rep. Ionos. Res. Space Res. Jap., 25, 163, 1971.

Mansurov, S. M., New evidence of a relationship between magnetic fields in space and on earth, Geomagn. Aeron., 9, 622, 1969.

Mansurov, S. M., and L. G. Mansurova, The relationship between the magnetic fields in space and at the earth's surface, Ann. Geophys., 26, 397, 1970. 
Svalgaard, L., Sector structure of the interplanetary magnetic field and daily variation of the geomagnetic field at high latitudes, Pap. 6, Dan. Meteorol. Inst. Geophys., Charlottenlund, Denmark, 1968.

Svalgaard, L., Interplanetary magnetic sector structure 1926-1971, Pap. 29, Dan. Meteorol. Inst. Geophys., Charlottenlund, Denmark, 1972.

Wilcox, J. M., and N. F. Ness, Quasi-stationary corotating structure in the interplanetary medium, J. Geophys. Res., 70, 5793, 1965.
Wilcox, J. M., and A. S. Tanenbaum, The influence of solar differential and rigid rotation on the interplanetary magnetic field, Space Sci. Lab. Rep., Ser. 12, issue 45, Univ. of Calif., Berkeley, June 1971.

Wilcox, J. M., K. H. Schatten, and A. S. Tanenbaum, Photospheric magnetic field rotation: Rigid and differential, Solar Phys., 14, 255, 1970.

(Received January 31, 1972; accepted April 26, 1972.) 